\title{
Digital speckle correlation for curved surfaces
}

\author{
Ichirou Yamaguchi* \\ Scientist Emeritus of RIKEN, Higashi-Hisakata 3-4-32, Kiryu, Gunma 376-0053 Japan
}

Received May 19, 2012; accepted June 25, 2012; published June 30, 2012

\begin{abstract}
Displacement of speckles arising in the diffraction field of a laser-illuminated surface subject to homogeneous deformation is investigated by numerical simulations using a rough surface model represented by an assembly of point scatterers distributed randomly around its mean (possibly arbitrary) surface. The results obtained from the simulation are compared with those derived from analytical approach that assumes a plane mean surface. These will be useful for designing and constructing displacement and deformation measurement systems using a digital correlation technique.
\end{abstract}

Laser speckles that arise from random interference of light scattered from a rough surface are highly contrasted and their size distributions are independent of microscopic structure if the surface roughness is larger than the wavelength and its correlation length is sufficiently small (so-called fully developed speckle). When the rough surface is subjected to displacement or deformation, speckles reveal the displacement and decorrelation that can be observed clearly in 3-dimentional space. Speckle displacement results from a change of phase relationship introduced by surface deformation or displacement. Speckle displacement is defined quantitatively by the peak position of the cross-correlation of speckle intensity distributions before and after object displacement or deformation and endows speckles with a role of clear fingerprints attached to the object. Hence speckle displacement is widely used for measurement of movement or deformation of rough objects by means of speckle photography and digital correlation technique. It is to be noticed, however, that the speckle displacement generally deviates from that of the surface depending on the sort of deformation and optical systems in a rather complicated manner. It also depends on the position of observation. Theoretical derivations of speckle displacement have been reported using geometrical optics and wave optics [1,2]. A general investigation on speckle displacement and decorrelation in the diffraction and the image fields was reported in the case of a plane surface subject to homogeneous deformation to be decomposed into translation, rotation, and strain with linear transmission of complex amplitude from a plane rough surface to an observation plane [1]. Speckle displacement also governs the contrast of fringes observed in holographic and speckle

${ }^{*}$ E-mail: ichiyama1115@yahoo.co.jp interferometry [3]. Although we often need to measure not only flat surfaces but also curved surfaces, speckle displacement caused by deformation of non planar surfaces has not been examined yet. In this paper we derive the distribution of 2-d speckle displacement from curved surfaces. Since an analytical approach becomes more complicated than in the case of a flat surface, we employ here a numerical simulation that is general and more flexible.

We examine speckle displacement in the diffraction field of a rough surface illuminated by a narrow laser beam. Speckle displacement in the field has been used for measurements of translation and strain $[4,5]$. The new approach can be applied to general conditions for speckle detection and its mathematics is simpler and more straightforward than analytical methods. We adopt the optical model of a rough surface that is represented by an assembly of point scatterers which are randomly distributed around its mean surface. We calculate a change in path differences of superposed scattered waves induced by object deformation.

We start from the coordinate system shown in Figure 1. Whereas in the previous paper describing speckle displacement in digital holography [6] we used a 2-dimensional version of the system, an extension to 3-dimension extension is employed here.

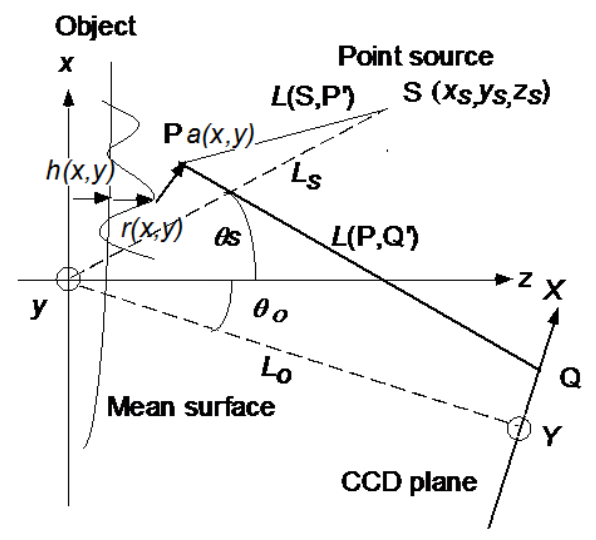

Fig. 1. Coordinate systems for simulation 
A rough object is illuminated by a point monochromatic source $S$ with the wave number $k=2 \pi / \lambda$. An object point $P[x, y, h(x, y)+r(x, y)]$ on an initial object where $h(x, y)$ and $r(x, y)$ represent the initial mean surface and the random roughness profile, respectively, is displaced to the point $P\left[x+a_{x}(x, y), \quad y+a_{y}(x, y), \quad h(x, y)+r(x, y)+a_{z}(x, y)\right]$ with the displacement vector $\boldsymbol{a}\left(a_{x}, a_{y}, a_{z}\right)$. The complex amplitudes at the CCD plane before and after object deformation are represented by the superpositions of the spherical waves scattered by assemblies of object point $P$, respectively. The distribution of surface roughness $r(x, y)$ is represented by the random numbers uniformly distributed between $\left(-r_{m} / 2\right.$, $r_{m} / 2$ ) where $r_{\mathrm{m}}$ represents the maximum surface roughness. At the observation plane the complex amplitudes arising from the surface point associated with a sample point $\left(x_{j}, y_{j}\right)$ at the object plane are added with macroscopic amplitude of the incident beam but with random phase shifts introduced by the surface roughness such as

$$
U(Q: \mathbf{a})=\sum_{j=1}^{M} \sqrt{I_{O}\left(x_{j}+a_{x j}, y_{j}+a_{y j}\right)} \exp \{i k[L(S: P)+L(P: Q)]\},
$$

where $I_{0}(x, y)$ is the macroscopic intensity distribution at the object and

$$
L(A: B)=\sqrt{\left(x_{A}-x_{B}\right)^{2}+\left(y_{A}-y_{B}\right)^{2}+\left(z_{A}-z_{B}\right)^{2}}
$$

is the distance between the points $\mathrm{A}\left(x_{\mathrm{A}}, y_{\mathrm{A}}, z_{\mathrm{A}}\right)$ and $\mathrm{B}\left(x_{\mathrm{B}}, \mathrm{y}_{\mathrm{B}}, z_{\mathrm{B}}\right)$. At the CCD plane whose origin is distant from the object by $L_{O}$ in the direction $\theta_{0}$ the complex amplitude from the point scatterers is combined with each other to be detected by a CCD. Each pixel of the CCD with the pitch $p$, the width $w$, and the pixel number $N x N$ delivers the output given by

$$
S(m, n: \mathbf{a})=\iint_{\text {pixel }} I(X, Y: \mathbf{a}) d X d Y \quad(m, n=1 \sim N)
$$

We can derive speckle displacement at the observation plane from the cross-correlation function of the video signal defined in terms of the total pixel number $N_{T}$ by

$$
C(M, N: \mathbf{a})=\sum_{m=1, n=1}^{N_{T}-N, N_{T}-N} S(m, n: 0) S(m+M, n+N: \mathbf{a}) / C(0,0: \mathbf{a}),
$$

whose peak position and height mean speckle displacement and decorrelation due to object deformation, respectively.

The above algorithm was programmed by using Mathematica 5.1. Although it allows to analyze speckle displacement caused by an arbitrary deformation, that is, an arbitrary distribution of the displacement vector, we assume here a homogeneous deformation of the illuminated area where object displacement can be approximated by the linear expansion represented by the following equations. $a_{x}(x, y)=a_{x 0}+\varepsilon_{x x} x-\left(\Omega_{z}-\varepsilon_{x y}\right) y$,

$a_{y}(x, y)=a_{y 0}+\left(\Omega_{z}+\varepsilon_{x y}\right) x+\varepsilon_{y y} y$,

$a_{z}(x, y)=a_{z 0}-\Omega_{y} x+\Omega_{x} y$,

where $\left(a_{\mathrm{x} 0}, a_{\mathrm{y} 0}, a_{\mathrm{z} 0}\right)$ and $\left(\Omega_{\mathrm{x}}, \Omega_{\mathrm{y}}, \Omega_{\mathrm{z}}\right)$, mean the translation along and about each axis, and $\left(\varepsilon_{\mathrm{xx}}, \varepsilon_{\mathrm{xy}}, \varepsilon_{\mathrm{yy}}\right)$ stands for in-plane strain coefficients, respectively. They are defined by the following equations

$$
\begin{aligned}
& \left(a_{x 0}, \quad a_{y 0}, \quad a_{z 0}\right)=\left[a_{x}(0,0), \quad a_{y}(0,0), \quad a_{z}(0,0)\right], \\
& \left(\Omega_{x}, \quad \Omega_{y}, \Omega_{z}\right)=\left\{\left(\frac{\partial a_{z}}{\partial y}\right)_{0},-\left(\frac{\partial a_{z}}{\partial x}\right)_{0}, \frac{1}{2}\left[\left(\frac{\partial a_{y}}{\partial x}\right)_{0}-\left(\frac{\partial a_{x}}{\partial y}\right)_{0}\right]\right\}, \\
& \varepsilon_{x x}=\left(\frac{\partial a_{x}}{\partial x}\right)_{0}, \quad \varepsilon_{y y}=\left(\frac{\partial a_{z}}{\partial y}\right)_{0}, \quad \varepsilon_{x y}=\varepsilon_{y x}=\frac{1}{2}\left[\left(\frac{\partial a_{y}}{\partial x}\right)_{0}+\left(\frac{\partial a_{x}}{\partial y}\right)_{0}\right] .
\end{aligned}
$$

Figure 2 shows an example of the simulated speckle patterns and their autocorrelation and cross-correlation. The rough surface which has a plane mean surface and the maximum roughness of $r_{m}=0.1 \mathrm{~mm}$ is illuminated normally with a parallel beam of $1 \mathrm{~mm}$ diameter with the wavelength of $657 \mathrm{~nm}$. A matrix CCD having the pixel pitch $p=5 \mu \mathrm{m}$, the aperture width $w=5 \mu \mathrm{m}$, and the pixel number $N_{T}=16$ along $\mathrm{X}$ and $\mathrm{Y}$ axes is placed at a distance of $L_{\mathrm{O}}=200 \mathrm{~mm}$ from the object in the direction of $\theta_{0}=30$ degrees. The number of sample points at the object plane is $16 \times 16$. The interference intensity resulting from superposition of this number of wavelets originating from the point scatterers is integrated over each pixel of the CCD. Figures 2(a)-(b) represent the detected patterns before and after in-plane translation $a_{x 0}=0.3 \mathrm{~mm}$. Each pattern is Fourier transformed and the conjugate product of the resultant spectra is calculated and is then subject to inverse Fourier transform to yield the cross-correlation function. The autocorrelation of (a) shown by Fig.2(c) has the peak at the center $M=N=8$, while the cross-correlation shown in (d) presents the peak around $M=14, N=8$ indicating speckle displacement. The peak position of the cross-correlation was derived from peak interpolation using the $3 \times 3$ pixel values around the maximum pixel. Next we present the results for in-plane and out-of-plane translations of curved surfaces.

Speckle displacement corresponds to the shift of the observation point where the random phase relationship caused by deformation is preserved. When this point remains stationary, speckles show no displacement as called speckle boiling in which only speckle decorrelation occurs. Fringe localization is observed in holographic interferometry at the plane of speckle boiling. 


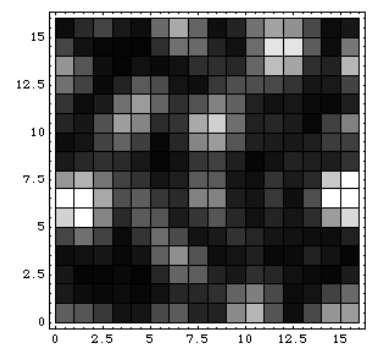

(a)

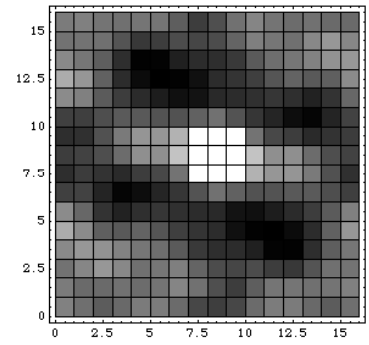

(c)

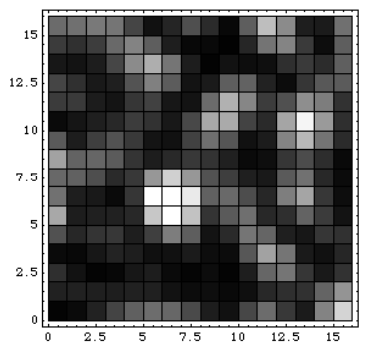

(b)

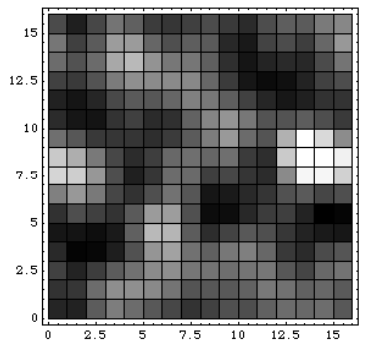

(d)
Fig. 2. Simulated speckle patterns recorded in the diffraction field before (a) and after (b) in-plane translation of $a_{\mathrm{x} 0}=0.3 \mathrm{~mm}$ of a plane rough surface, its autocorrelation (c) and cross-correlation (d).

Analytical expressions of speckle displacement which were derived by considering the linear transmission of complex amplitude from the object to an observation plane in the diffraction field [2] are given as functions of translation, rotation, and strain coefficients. Here we show only the terms proportional to translation which are given by

$$
\begin{aligned}
& A_{X}=a_{x 0}\left(\frac{L_{O} \cos ^{2} \theta_{S}}{L_{S} \cos \theta_{O}}+\cos \theta_{O}\right)-a_{z 0}\left(\frac{L_{O} \cos \theta_{S} \sin \theta_{S}}{L_{S} \cos \theta_{O}}+\sin \theta_{O}\right), \\
& A_{Y}=a_{y 0}\left(\frac{L_{O}}{L_{S}}+1\right) .
\end{aligned}
$$

The results of simulation for spherical surfaces are compared with these equations derived for a flat surface. The observation plane is located at a distance of $200 \mathrm{~mm}$ from the object in the direction of $\theta_{0}=30$ degrees as in the case of in-plane translation. The initial object mean surface is represented by $h(x, y)=-\left(x^{2}+y^{2}\right) / 2 R$ with the radius of curvature $R$.

Figure 3 (a) represents the dependence of the ratio of the evaluated displacement sensitivity $A_{X} / a_{x O}$ for in-plane translation to that obtained from Eq.(6) on the radius of the object surface curvature, while Figure 3(b) means the same dependence of $A_{X} / a_{z O}$ for out-of-plane translation. The standard deviation of each ratio value calculated from 8 simulations with different sets of random numbers were less than $0.5 \%$. The displacement sensitivities for in-plane and out-of-plane translations of a flat surface are equal to 0.867 and -0.5 , respectively. We notice no remarkable dependence of speckle displacement sensitivities due to both in-plane and out-of-plane translation on the surface curvature.
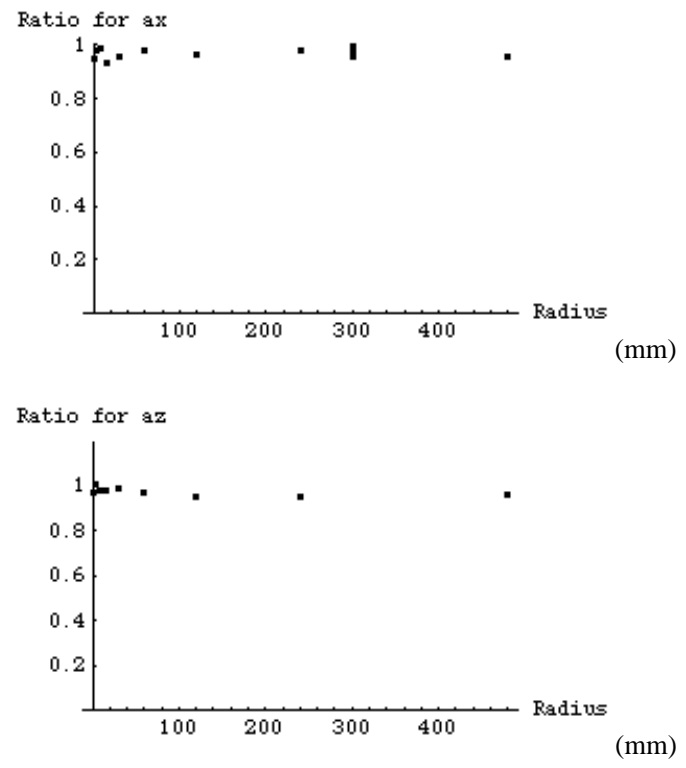

Fig. 3. Dependences of speckle displacement sensitivity on the curvature radii of the surface for (a) in-plane and (b) out-of-plane translation.

In this paper we have described a numerical simulation of speckle displacement caused by homogeneous deformation of a curved surface illuminated by a narrow laser beam. We employed a model of a rough surface that is based on an assembly of point scatterers distributed randomly about its mean surface. The dependences of speckle displacement sensitivity for in-plane and out-of plane object translation on surface shape have been investigated for a plane and a sphere mean surface. The results for a plane surface agree well with those calculated by the analytical approach published previously. The present approach also enables to predict statistical behaviors of the digital speckle correlation technique that seriously affects the measurement accuracy in practice

\section{References}

[1] P. Jacquot, P.K. Rastogi, Appl. Opt. 18, 2022 (1979).

[2] I. Yamaguchi, Optica Acta 28, 1359 (1981).

[3] I. Yamaguchi, Opt. Lasers in Eng. 39, 411 (2003).

[4] I. Yamaguchi, J. Phys. E 19, 944 (1986).

[5] I. Yamaguchi, T. Takemori, K. Kobayashi, Opt. Engin. 32(3), 618 (1993).

[6] I. Yamaguchi, Chinese Opt. Lett. 7, 1104 (2009). 